\title{
Electron Microscopy of Suspicious Samples and Infectious Specimens: Research and Diagnostics
}

\author{
D.R. Beniac and T.F. Booth
}

National Microbiology Laboratory, 1015 Arlington Street, Winnipeg, Manitoba, R3E 3R2, Canada

One of the key considerations in rapid diagnostics of infectious materials is the ability to accurately identify a specimen, and provide optimal protection for the staff performing the tests. In the event of a disease outbreak, or an intentional release of a bioagent the results of these tests are extremely time sensitive. Nucleic acid based procedures are rapid and extremely accurate; however additional information on the structural and chemical composition of an agent can be crucial in the identification process. Negative staining and subsequent observation in the transmission electron microscope (TEM) is the primary diagnostic test in the electron microscopy lab [1]. This method has the benefit of being extremely rapid and is capable of examining a wide range of samples with varied sizes, that can be biological or inorganic in origin. In the event that a suspicious sample is being investigated, the chemical composition of the specimen should also be ascertained using a technique such as energy dispersive spectroscopy. Brewer et al., [2] utilized microanalysis techniques in the chemical characterization of simulated bioagents. In addition, scanning electron microcopy (SEM) can also be extremely beneficial in the identification of unknown fluid biological samples, and dry powder samples.

When working with a novel pathogen, both diagnostics and research can be extremely time sensitive. Often once the diagnostic "phase" of the work is completed, it is followed by an intensive research program to help better understand the novel pathogen being studied. Recent technical advances in the field of electron microscopy have drastically shortened the turn around time in cryo-electron microscopy (cryo-EM) and tomography (cryo-ET) procedures. Improvements have included; stable cryo-stages, electronic detectors replacing photographic film, software advances which have automated data collection in cryo-EM and cryo-ET, automated freeze plunge devices, commercially available perforated support films with regularly spaced holes, and the overall improvements in computer technology and software which analyze electron microscopy data. The combination of all of these improvements has made cryo-EM and cryo-ET useful tools for research of infectious specimens $[3,4]$.

In summary, the challenges that face a diagnostic EM virology laboratory are; rapid detection, structural preservation, and the appropriate fixation or inactivation for BSL2, BSL3, and BSL4 pathogens. These initial rapid detection procedures are best achieved by TEM/negative staining, or SEM/sputter coating. This work can then be followed by subsequent investigations using alternative techniques which can include cryo-EM, cryo-ET, and image analysis procedures.

\section{References}

[1] M. Laue and N. Bannert. J Applied Microbiology, 109, 1159-1168, (2010).

[2] L.N. Brewer et al., Forensic. Sci. Int., 179, 98-106, (2008).

[3] D.R. Beniac et al., Nat. Str. Mol. Biol. 13, 751-752, (2006)

[4] D.R. Beniac et al., PLoS One. 7(1): e29608. doi:10.1371/journal.pone.0029608, 1-12, (2012). 

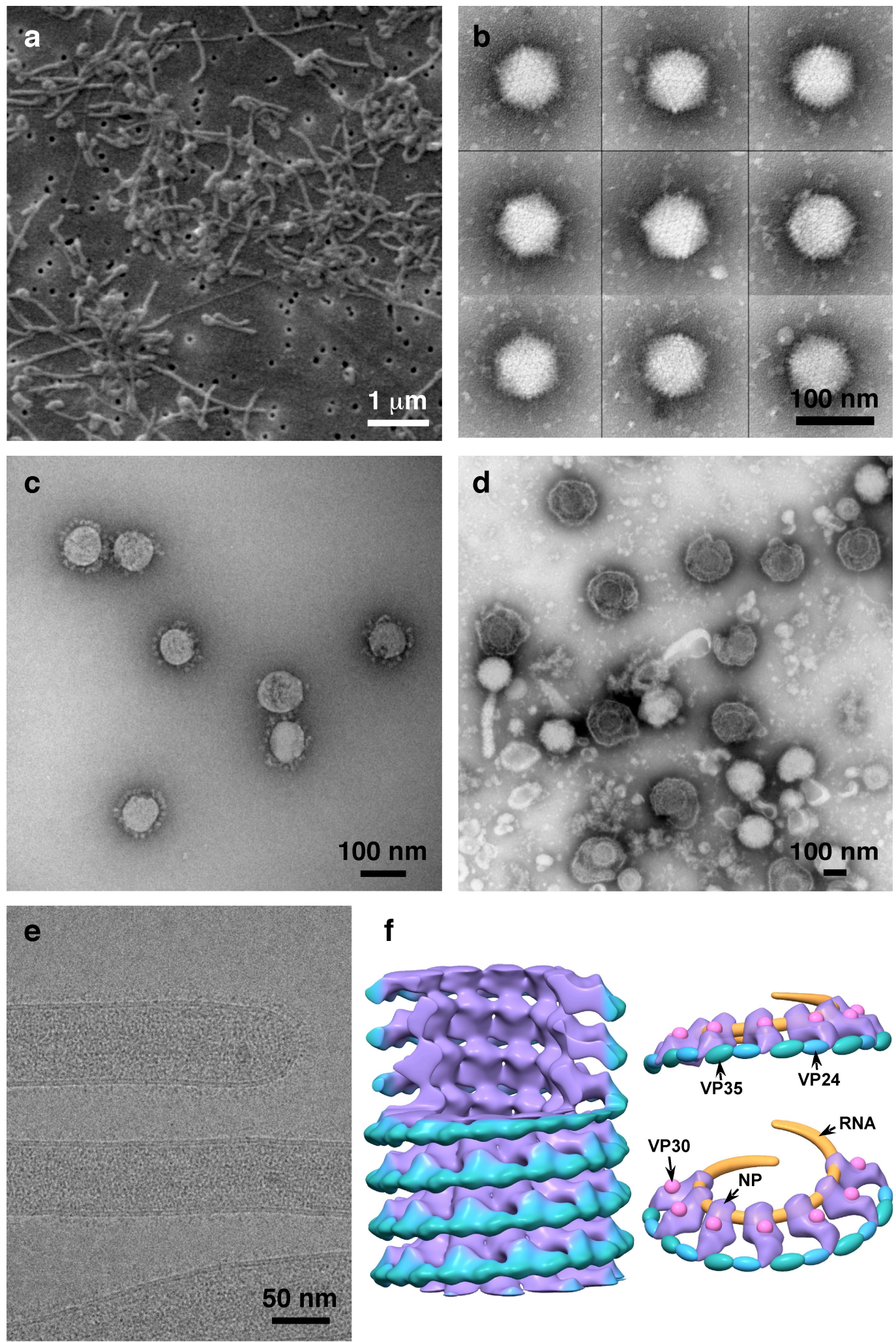

f
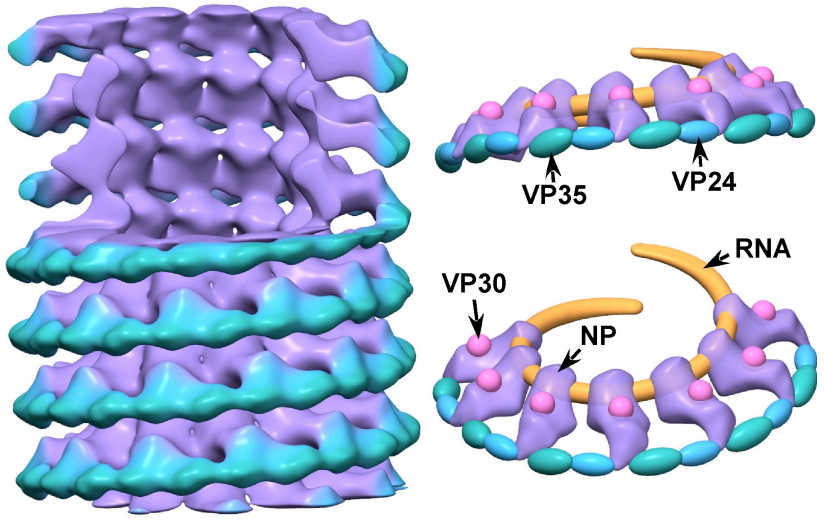

Electron microscopy of viruses: (a) SEM image of gold sputter coated Ebola virus. (b) Negative stained TEM images of Adenovirus (c) Negative stained TEM image of gradient purified SARS-CoV. (d) Negative stained TEM image of Herpesvirus direct from tissue culture (e) Cryo-EM image of Ebola virus, no stain used (f) Cryo-EM 3D reconstruction of Ebola virus nucleocapsid. 